УДК 548.1

\title{
СИСТЕМАТИКА РЕШЕТОК СОВПАДАЮЩИХ УЗЛОВ ДЛЯ ОЦК И ГЦК КРИСТАЛЛОВ
}

\author{
(c) 2018 Б. М. Даринский, Н. Д. Ефанова, А. С. Прижимов \\ Воронежский государственный университет, Университетская пл., 1, 394018 Воронеж, Россия \\ e-mail:darinskii@mail.ru
}

Поступила в редакцию 15.10.2018

\begin{abstract}
Аннотация. В настоящей работе предложена новая методика построения решетки совпадающих узлов для ОЦК и ГЦК кристаллов. Получены условия образования орторомбической, тетрагональной и кубической решетки совпадений и указаны конкретные решетки этих сингоний. Показаны возможные поликристаллические структуры, имеющие общую решетку совпадающих узлов.
\end{abstract}

Ключевые слова: решетка совпадающих узлов, кристалл, разориентация, межфазные границы, структура.

DOI: https://doi.org/10.17308/kcmf.2018.20/632

\section{ВВЕДЕНИЕ}

Специальные границы между кристаллитами выделяются из всей совокупности межкристаллитных границ, прежде всего, из-за их повышенной устойчивости, что связано с их пониженной энергией. Интерес к этим границам продолжается от первых работ шестидесятых годов прошлого века [2-6] по настоящее время [7-17]. Важным направлением, связанным со знаниями в области структуры и свойств межкристаллитных границ и возникшим в последние два десятилетия, является целенаправленное приготовление поликристаллов, имеющих заданную геометрию размещения отдельных кристаллитов и межкристаллитных границ (Grain Boundary Engineering) как в трехмерных [18, 19], так и в пленочных образцах [20]. Надежды на успех в решении этих задач основаны на существенном развитии методов направленной кристаллизации, в частности, в наноразмерных материалах [19]. Поэтому следует полагать, что исследование строения границ и их совокупности в поликристаллических образцах является актуальным. В предыдущей работе авторов [1] была развита методика, позволяющая классифицировать совокупность специальных границ в кристаллах, имеющих простую кубическую решетку. В настоящей работе аналогичная задача решается для ОЦК и ГЦК кристаллов, указываются условия формирования решеток совпадения различных сингоний и совокупность параметров разориентации кристаллитов, имеющих общую решетку совпадающих узлов.

\section{РЕЗУЛЬТАТЫ И ИХ ОБСУЖДЕНИЕ}

Операция поворота твердого тела охарактеризуется векторами $\vec{B}_{0}, \vec{B}$ и $\vec{A}[1]$. Здесь векторы $\vec{B}_{0}$, $\vec{B}$ являются решеточными векторами, принадлежащими общей координационной сфере и поэтому имеющие одинаковые длины. Вектор $\vec{A}$ является осью вращения, переводящего $\vec{B}_{0}$ в $\vec{B}$. Векторы $\vec{B}_{0}$ и $\vec{B}$ выбираются произвольно, а вектор $\vec{A}$ лежит в кристаллографической плоскости, перпендикулярной вектору $\vec{B}-\vec{B}_{0}$. Такая плоскость определяется базисными векторами:

$$
\vec{P}_{1}=\left[\vec{B} \vec{B}_{0}\right], \vec{P}_{2}=\vec{B}+\vec{B}_{0},
$$

Отметим, что $\vec{P}_{1}$ совпадает по направлению с вектором обратной решетки, построенным на векторах $\vec{B}$ и $\vec{B}_{0}, \vec{P}_{2}$ лежит на биссектрисе этих векторов. После проведения операций (1) $\vec{B}_{0}=m_{1} \vec{a}_{1}+m_{2} \vec{a}_{2}+m_{3} \vec{a}_{3}$, возможно, следует сократить результат, так, чтобы векторы $\vec{P}_{1}$ и $\vec{P}_{2}$ стали минимальными решеточными векторами. Совокупность всех осей вращения определяется выражением:

$$
p_{1} \vec{P}_{1}+p_{2} \vec{P}_{2}
$$

где $p_{1}, p_{2}$ - целые и полуцелые числа, выбор которых позволяет однозначно определить вектор $\vec{A}$.

Угол поворота $\varphi$ определяется следующей формулой для $\cos \varphi[1]$ :

$$
\begin{gathered}
\cos (\varphi)=\frac{\left(\overrightarrow{B_{0}} \vec{B}\right) A^{2}-(\vec{A} \vec{B})^{2}}{B^{2} A^{2}-(\vec{A} \vec{B})^{2}}, \\
\vec{B}=n_{1} \vec{b}_{1}+n_{2} \vec{b}_{2}+n_{3} \vec{b}_{3},
\end{gathered}
$$


где $n_{i}, m_{i}$ - целые числа для примитивной кубической решетки и целые и полуцелые для объемно- и гранецентрированной, а $\vec{a}_{i}-$ вектора ячеек этих кристаллических решеток. В нашем случае вектоpa $\vec{a}_{i}$ будут совпадать по направлениям с ортами $\vec{i}, \vec{j}, \vec{k}$ декартовой системы координат и выражаться через них следующим образом:

$$
\vec{a}_{1}=a \vec{i}, \vec{a}_{2}=a \vec{j}, \vec{a}_{3}=a \vec{k},
$$

где $a$ - длина ребра кубической ячейки, в дальнейшем она будет считаться равной 1.

В качестве третьего вектора решетки совпадений выберем вектор:

$$
\vec{C}=[\vec{A} \vec{B}] .
$$

Зная вектор $\vec{C}$, можно записать число атомов $\Sigma$ в ячейке решетки совпадений:

$$
\Sigma=\vec{B} \vec{A} \vec{C}=B^{2} A^{2}-(\vec{A} \vec{B})^{2} .
$$

Число пар кристаллографических векторов, которые можно совместить путем поворота, задается количеством узлов в координационной сфере, к которой относятся начальный и конечный векторы.

В работе [1] построена систематика решеток совпадений для случая примитивной кубической решетки и рассмотрены их различные варианты. Показано, что в общем случае решетка совпадений будет иметь моноклинную сингонию, и указаны некоторые частные случаи, когда решетка совпадающих узлов может обладать более высокой симметрией: орторомбической, тетрагональной или кубической.

В настоящей работе сформулированы условия получения тетрагональной и кубической структур. Пусть векторы $\vec{B}$ и $\vec{B}_{0}$ перпендикулярны и принадлежат одной координационной сфере, следовательно, их компоненты можно записать двумя разными способами:

$$
\vec{B}_{0}=[\operatorname{lmn}], \vec{B}=[m l \bar{n}], \vec{B}_{0}=[\operatorname{lmn}], \vec{B}=[m n \bar{l}] .
$$

Рассмотрим первый случай, для него условием перпендикулярности $\vec{B}$ и $\vec{B}_{0}$ будет равенство:

$$
2 m l=n^{2} .
$$

Пусть $m=2 k^{2}, l=s^{2}$, тогда $n$ принимает значение $2 k s$, где $k$ и $s-$ произвольные положительные целые числа. Тогда семейство векторов $\vec{B}$ и $\vec{B}_{0}$ можно представить в виде:

$$
\vec{B}_{0}=\left[s^{2}, 2 k^{2}, 2 k s\right], \vec{B}=\left[2 k^{2}, s^{2},-2 k s\right] .
$$

Выбирая различные значения $k$ и $s$, мы находим различные значения $\vec{A}$ и $\vec{C}$ и выбираем из них те, которые удовлетворяют условиям тетрагональ- ной структуры $(|\vec{B}|=|\vec{C}|$ или $|\vec{B}|=|\vec{A}|)$ или кубической $(|\vec{B}|=|\vec{C}|=|\vec{A}|)$.

Рассмотрим второй случай, для него условием перпендикулярности $\vec{B}$ и $\vec{B}_{0}$ будет равенство: $l m+m n=n l$, из которого выражаем:

$$
n=\frac{l m}{l-m} \text {. }
$$

Положим $l=m+k$, а $m=k s$, тогда $n=s(k s+k)$. Отсюда, вектора $\vec{B}_{0}$ и $\vec{B}$ после отбрасывания общего множителя принимают следующий вид:

$$
\vec{B}_{0}=[s+1, s, s(s+1)], \vec{B}=[s, s(s+1),-(s+1)] .
$$

Это свидетельствует о существовании однопараметрического семейства векторов $\vec{B}_{0}$ и $\vec{B}$, которые порождают в общем случае тетрагональную решетку совпадающих узлов.

Приведем ниже табл. 1, содержащую примеры тетрагональной и кубической структур решеток совпадений, имеющих наименьшие величины постоянных решеток.

Рассмотрим ОЦК решетку. Возьмем вектор $\vec{B}_{0}$ в виде:

$$
\vec{B}_{0}=[l+1 / 2, m+1 / 2, n+1 / 2]
$$

Вектор $\vec{B}$ будет иметь такие же компоненты, которые переставлены местами и могут иметь противоположный знак. В общем случае будет опять получаться моноклинная структура решетки совпадающих узлов. Однако в этом случае вектора этой решетки будут иметь полуцелые или целые компоненты. Возьмем для примера вектор $\vec{B}_{0}=\left[\frac{3}{2} \frac{1}{2} \frac{1}{2}\right]$, а вектор $\vec{B}=\left[\frac{1}{2} \frac{3}{2} \frac{1}{2}\right]$. Определяем вид вектора $\vec{A}: \vec{A}=\left[p_{2}-p_{1}, p_{1}-p_{2}, 2 p_{1}+p_{2}\right]$. Если взять $p_{2}=p_{1}=1$, то вектор $\vec{A}=[001]$, и, зная $\vec{A}$ и $\vec{B}$, определяем $\vec{C}=[3 \overrightarrow{1} 0]$. Условия и методика определения орторомбической структуры решетки совпадений такие же, что и в случае примитивной решетки. Приведем ниже табл. 2 примеров для моноклинной и орторомбической структур решетки совпадений в случае ОЦК решетки исходного кристалла.

Проанализируем случай ГЦК решетки. Возьмем вектор $\vec{B}_{0}$ в виде:

$$
\vec{B}_{0}=[l+1 / 2, m+1 / 2, n] .
$$

Вектор $\vec{B}$ будет иметь такие же компоненты, которые переставлены местами и могут иметь противоположный знак. Решетка совпадений, если исходная решетка - ГЦК, имеет в общем случае также моноклинную структуру. Отличие бу- 


\section{СИСТЕМАТИКА РЕШЕТОК СОВПАДАЮЩИХ УЗЛОВ ДЛЯ ОЦК И ГЦК КРИСТАЛЛОВ}

Таблица 1. Характеристики угла поворота и ячейки решетки совпадающих узлов тетрагональной и кубической структур.

[Table 1. Characteristics of the lattice cell of matching nodes and the angle of rotation of tetragonal and cubic structures]

\begin{tabular}{|c|c|c|c|c|c|c|c|c|c|}
\hline $\mathbf{B}_{0}$ & $\mathbf{B}$ & $p_{1}$ & $p_{2}$ & $\mathbf{P}_{1}$ & $\mathbf{P}_{2}$ & $\mathbf{A}$ & $\mathbf{C}$ & $\varphi$ & $\Sigma$ \\
\hline \multicolumn{7}{|c|}{ Тетрагональная структура [Tetragonal structure] } \\
\hline$[81 \overline{4}]$ & {$[184]$} & 1 & 0 & {$[\overline{4} 47]$} & {$[110]$} & {$[\overline{4} 47]$} & {$[81 \overline{4}]$} & $90^{\circ}$ & 6561 \\
\hline$[29 \overline{6}]$ & {$[926]$} & 1 & 0 & {$[\overline{6} 67]$} & {$[110]$} & {$[\overline{6} 67]$} & {$[29 \overline{6}]$} & $90^{\circ}$ & 14641 \\
\hline$[9812]$ & {$[89 \overline{12}]$} & 1 & 0 & {$[12 \overline{12} 1]$} & {$[110]$} & {$[12 \overline{12} 1]$} & {$[9812]$} & $90^{\circ}$ & 83521 \\
\hline \multicolumn{8}{|c|}{ Кубическая структура [Cubic structure] } \\
\hline$[122]$ & {$[21 \overline{2}]$} & 1 & 0 & {$[2 \overline{2} 1]$} & {$[110]$} & {$[2 \overline{2} 1]$} & {$[122]$} & 90 & 81 \\
\hline$[632]$ & {$[\overline{3} 26]$} & 1 & 0 & {$[\overline{2} 6 \overline{3}]$} & {$[358]$} & {$[\overline{2} 6 \overline{3}]$} & {$[632]$} & 90 & 2401 \\
\hline$[1243]$ & {$[\overline{4} 31]$} & 1 & 0 & {$[\overline{3} 12 \overline{4}]$} & {$[8715]$} & {$[\overline{3} 12 \overline{4}]$} & {$[1243]$} & 90 & 28561 \\
\hline
\end{tabular}

Таблица 2. Характеристики угла поворота и ячейки решетки совпадающих узлов моноклинной и орторомбической структур для ОЦК решетки.

[Table 2. Characteristics of the lattice cell of matching nodes and the angle of rotation of monoclinic and orthorhombic structures for bcc lattice]

\begin{tabular}{|c|c|c|c|c|c|c|c|c|c|}
\hline $\mathbf{B}_{0}$ & $\mathbf{B}$ & $p_{1}$ & $p_{2}$ & $\mathbf{P}_{1}$ & $\mathbf{P}_{2}$ & $\mathbf{A}$ & $\mathbf{C}$ & $\varphi$ & $\Sigma$ \\
\hline \multicolumn{10}{|c|}{ Моноклинная структура [Monoclinic structure] } \\
\hline 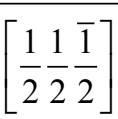 & {$\left[\begin{array}{lll}\overline{1} & \frac{1}{2} & \frac{1}{2}\end{array}\right]$} & 1 & 2 & [011] & [011] & {$[0 \overline{3} 5]$} & [253] & $142.14^{\circ}$ & 19 \\
\hline$\left[\frac{1}{2} \frac{3}{2} \frac{1}{2}\right]$ & {$\left[\frac{3}{2} \frac{1}{2} \frac{1}{2}\right]$} & 1 & 1 & [114] & {$[22 \overline{1}]$} & [552] & {$\left[\begin{array}{lll}11 & 1 & 0\end{array}\right]$} & $78.46^{\circ}$ & 135 \\
\hline$\left[\frac{3}{2} \frac{3}{2} \frac{1}{2}\right]$ & {$\left[\begin{array}{lll}\frac{3}{2} & 1 & \frac{3}{2} \\
\frac{3}{2}\end{array}\right]$} & 1 & 1 & {$[2 \overline{3} \overline{3}]$} & [011] & {$[2 \overline{15}]$} & {$\left[\begin{array}{llll}8 & 2 & 1 & 1\end{array}\right]$} & $101.72^{\circ}$ & 253 \\
\hline \multicolumn{10}{|c|}{ Орторомбическая структура [Orthorhombic structure] } \\
\hline$\left[\frac{1}{2} \frac{3}{2} \frac{1}{2}\right]$ & 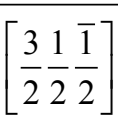 & 1 & 0 & [114] & [22ī] & [114] & {$\left[\begin{array}{lll}5 & 13 & 2\end{array}\right]$} & $50.48^{\circ}$ & 99 \\
\hline$\left[\begin{array}{lll}3 & \frac{3}{2} & \frac{1}{2}\end{array}\right]$ & {$\left[\begin{array}{lll}\frac{3}{2} & \frac{1}{2} & \frac{3}{2}\end{array}\right]$} & 1 & 0 & {$[2 \overline{33}]$} & [011] & {$[2 \overline{33}]$} & {$\left[\begin{array}{lll}12 & 15 & \overline{7}\end{array}\right]$} & $99.08^{\circ}$ & 209 \\
\hline$\left[\frac{1}{2} \frac{5}{2} \frac{\overline{1}}{2}\right]$ & {$\left[\begin{array}{lll}\frac{5}{2} & \frac{1}{2} & \frac{1}{2}\end{array}\right]$} & 1 & 0 & 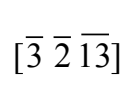 & {$[\overline{2} 30]$} & 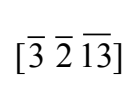 & {$\left[\begin{array}{llll}1 & 68 & \overline{13}\end{array}\right]$} & $92.12^{\circ}$ & 2457 \\
\hline
\end{tabular}

дет заключаться лишь в виде векторов, образующих ее. Возьмем для примера вектор $\vec{B}_{0}=\left[\frac{3}{2} \frac{\overline{1}}{2} 0\right]$, а вектор $\vec{B}=\left[0 \frac{\overline{3}}{2} \frac{1}{2}\right]$. Определяем вид вектора $\vec{A}$ : $\vec{A}=\left[\frac{3}{2} p_{2}-\frac{1}{4} p_{1}, \frac{3}{4} p_{1}-\frac{1}{2} p_{2}, \frac{9}{4} p_{1}+\frac{1}{2} p_{2}\right]$.Если взять $p_{2}=p_{1}=1$, то вектор $\vec{A}=[517]$. Зная $\vec{A}$ и $\vec{B}$, опреде- ляем $\vec{C}=\left[11 \frac{\overline{5}}{2} \frac{\overline{15}}{2}\right]$. Условия и методика определения орторомбической структуры решетки совпадений такие же, что и в случае примитивной решетки, поэтому можно опустить их описание. Приведем ниже табл. 3 примеров для моноклинной и орторомбической структур решетки совпадений в случае ГЦК решетки исходного кристалла. 
Таблица 3. Характеристики угла поворота и ячейки решетки совпадающих узлов моноклинной и орторомбической структур для ГЦК решетки.

[Table 3. Characteristics of the lattice cell of matching nodes and the angle of rotation of monoclinic and orthorhombic structures for fcc lattice]

\begin{tabular}{|c|c|c|c|c|c|c|c|c|c|}
\hline $\mathbf{B}_{0}$ & $\mathbf{B}$ & $p_{1}$ & $p_{2}$ & $\mathbf{P}_{1}$ & $\mathbf{P}_{2}$ & $\mathbf{A}$ & $\mathbf{C}$ & $\varphi$ & $\Sigma$ \\
\hline \multicolumn{10}{|c|}{ Моноклинная структура [Monoclinic structure] } \\
\hline$\left[0 \frac{1}{2} \frac{1}{2}\right]$ & {$\left[\frac{1}{2} 0 \frac{\overline{1}}{2}\right]$} & 1 & 1 & [1111] & [110] & [311] & {$\left[\frac{\overline{1}}{2} 2 \frac{\overline{1}}{2}\right]$} & 146.44 & 18 \\
\hline$\left[\frac{1}{2} \frac{3}{2} 0\right]$ & {$\left[0 \frac{1}{2} \frac{3}{2}\right]$} & 1 & 1 & {$[\overline{9} 3 \overline{1}]$} & [243] & {$\left[\begin{array}{lll}7 & 11 & 5\end{array}\right]$} & {$\left[14 \frac{21}{2} \frac{\overline{7}}{2}\right]$} & 94.10 & 1274 \\
\hline$\left[0 \frac{3}{2} \frac{3}{2}\right]$ & {$\left[\frac{\overline{3}}{2} \frac{\overline{3}}{2} 0\right]$} & 1 & 1 & [111] & {$\left[\frac{\overline{3}}{2} 0 \frac{3}{2}\right]$} & [531] & {$\left[\frac{1}{2} \frac{1}{2} 4\right]$} & 126.22 & 5346 \\
\hline \multicolumn{10}{|c|}{ Орторомбическая структура [orthorhombic structure] } \\
\hline$\left[0 \frac{1}{2} \frac{3}{2}\right]$ & {$\left[\frac{1}{2} \frac{\overline{3}}{2} 0\right.$} & 1 & 0 & {$[\overline{9} 31]$} & {$[123]$} & {$[\overline{9} 3 \overline{1}]$} & {$\left[\frac{\overline{3}}{2} \frac{1}{2} 15\right]$} & 107.46 & 910 \\
\hline$\left[0 \frac{1}{2} \frac{5}{2}\right]$ & {$\left[\begin{array}{lll}\overline{5} & \frac{1}{2} & 0\end{array}\right.$} & 1 & 0 & {$[\overline{1} 5 \overline{1}]$} & {$[\overline{5} 05]$} & [15ī] & {$\left[\frac{1}{2} \frac{\overline{5}}{2} 13\right]$} & 92.20 & 702 \\
\hline$\left[0 \frac{3}{2} \frac{5}{2}\right]$ & {$\left[\frac{3}{2} 0 \frac{\overline{5}}{2}\right]$} & 1 & 0 & [553] & [330] & [553] & {$\left[\frac{25}{2} 17 \frac{15}{2}\right]$} & 137.33 & 2006 \\
\hline
\end{tabular}

\section{ЗАКЛЮЧЕНИЕ}

В заключении сформулируем основные результаты работы. Найдены условия получения решеток совпадения различных сингоний в примитивных, ГЦК и ОЦК кубических кристаллах. Представлены таблицы для решеток совпадения с параметрами ячейки, имеющих малые индексы. Наличие разных сингоний решеток совпадения предполагает существование элементов симметрии для решеток совпадений, не являющихся элементами симметрии для контактирующих кристаллов. Количество таких элементов симметрии определяется группой симметрии решетки совпадения. Поэтому появляется возможность получения поликристаллических структур, имеющих разные углы разориентации кристаллитов на базе общей решетки совпадения. В таких поликристаллических структурах будут отсутствовать механические напряжения, связанные с наличием дисклинаций, вызванных несоответствием ориентаций контактирующих кристаллитов с огранкой каждого кристаллита специальными границами, и, как следствие, они будут иметь относительно высокую стабильность.

\section{СПИСОК ЛИТЕРАТУРЫ}

1. Даринский Б. М., Прижимов А. С., Шаров М. К. // Конденсированные среды имежфазные гранищь, 2018, т. 20 , № 1 , c. $50-55$. DOI: https://doi.org/10.17308/ kcmf.2018.20/476

2. Bollmann W. // Phil. Mag., 1967, vol. 16, pp. 363381. DOI: https://doi.org/10.1080/14786436708229748

3. Bollmann W. // Phil. Mag., 1967, vol. 16, pp. 383399. DOI: https://doi.org/10.1080/14786436708229749

4. Grimmer H. // Acta Cryst., 1974, vol. A 30, pp. 680680. DOI: https://doi.org/10.1107/S056773947400163X

5. Grimmer H., Bollmann W., Warrington D. T. // Acta Cryst., 1974, vol. A 30, pp. 197-207. DOI: https://doi. org/10.1107/S056773947400043X

6. Орлов А. Н., Перевезенцев В. Н., Рыбин В. В. Границы зерен в металлах. М.: Металлургия, 1980, $224 \mathrm{c}$.

7. Глейтер Г., Чалмерс Б. Большеугловые гранищы зерен. М.: Мир, 1975, 376 с.

8. Страумал Б. Б., Швиндлерман Л. С. // Поверхность. Физика, химия, механика, 1986, т. 10, с. 5-14.

9. Fortes M. A. // Phys. Stat. Sol., 1977, vol. B 82. pp. 377-382. DOI: https://doi.org/10.1002/pssb.2220820143

10. Bonnet R., Durand F. // Scripta Met., 1975, vol. 9, pp. 935-939. DOI: https://doi.org/10.1016/00369748(75)90548-7

11. Bonnet R. // Scripta Met., 1976, vol. 10, pp. 801806. DOI: https://doi.org/10.1016/0036-9748(76)90297-0

12. Bonnet R., Cousineau E. // Acta Cryst., 1977, vol. A 33, pp. 850-856. DOI: https://doi.org/10.1107/ S0567739477002058

13. Рыбин В. В., Перевезенцев В. Н. // ФТТ, 1975, T. 17, c. $3188-3193$. 
14. Андреева А. В., Фионова Л. К. // ФММ, 1977, т. 44 , с. $395-400$.

15. Кайбышев О. А., Валиев Р. З. Границы зерен и свойства металлов. М.: Металлургия, 1987, 214 с.

16. Копецкий Ч. В., Орлов А. Н., Фионова Л. К. Границьв зерен в чистых материалах. М.: Наука, 1987, $160 \mathrm{c}$.
17. Бокштейн Б. С. Структура и свойства внутренних поверхностей раздела в металлах. М.: Металлургия, 1988, 272 с.

18. Kobayashi S., Tsurekawa S., Watanabe T. // Beilstein J Nanotechnol, 2016, vol. 7, pp. 1829-1849.

19. Сухомлин Г. Д. // Металлофизика, новейшие технологии, 2013, т. 35, № 9, с. 1237-1249

20. Watanabe T. // Journal of Materials Science, 2011, vol. 46, pp. 4095-4115. DOI: https://doi.org/10.1007/ s10853-011-5393-z

\title{
SYSTEMATICS OF COINCIDENCE SITE LATTICES FOR BCC AND FCC CRYSTALS
}

\author{
(C) 2018 B. M. Darinsky, N. D. Efanova, A. S. Prizhimov \\ Vorornezh State University, 1, Universitetskaya pl., 394018 Voronezh, Russia \\ e-mail: darinskii@mail.ru
}

Received 15.10.2018

\begin{abstract}
The object of this study is polycrystalline materials containing intercrystalline boundaries in face centered cubic and body centered cubic crystals. A wide variety of geometric characteristics that determine the atomic structure and physical properties of intercrystalline boundaries, gives rise to the expediency of systematization of the entire set of possible boundaries. The intercrystalline boundaries obtained on the basis of the coincidence of nodes are an important family of defects in polycrystals, so these defects are given a lot of research, but currently there is no complete systematization. The construction of such systematization was the purpose of this paper.

The basis of classification is the coordination polyhedron of the face centered cubic and body centered cubic lattice. As parameters defining the geometry of the lattice matching of the nodes of the selected pair of vectors and the lattice vector that defines the axis of rotation was used. Using algebraic methods used in studies of solid rotation, formulas are obtained for the crystallographic plane in which all possible axes of turns and vectors of the lattice cell of matching nodes should lie. As a result, it was shown that the coincidence lattices of general form belong to monoclinic crystal structure. In particular cases they may belong to cubic crystal structure. It is shown that such lattices of coinciding nodes are possible, which are obtained at different parameters of rotation, so polycrystals on the basis of the common lattice of coincidences are possible. The tables for face centered cubic and body centered cubic crystals is constructed in which the data are systematized for the vectors of the cell of the lattice of coincidences, rotation angles, the number of atoms in the cell at different initial characteristics of rotation.
\end{abstract}

Keywords: lattice matching of the nodes of the crystal, disordering, interfaces, structure.

DOI: https://doi.org/10.17308/kcmf.2018.20/632

\section{REFERENCES}

1. Darinsky B. M., Prizhimov A. S., Sharov M. K. Condensed Matter and Interphases, 2018, vol. 20, no. 1, pp. 50-55. DOI: https://doi.org/10.17308/kcmf.2018.20/476 (in Russ.)

2. Bollmann W. Phil. Mag., 1967, vol. 16, pp. 363-381. DOI: https://doi.org/10.1080/14786436708229748

3. Bollmann W. Phil. Mag., 1967, vol. 16, pp. 383-399. DOI: https://doi.org/10.1080/14786436708229749
4. Grimmer H. Acta Cryst., 1974, vol. A 30, pp. 680680. DOI: https://doi.org/10.1107/S056773947400163X

5. Grimmer H., Bollmann W., Warrington D. T. Acta Cryst., 1974, vol. A 30, pp. 197-207. DOI: https://doi. org/10.1107/S056773947400043X

6. Orlov A. N., Perevezentsev V. N., Rybin V. V. Grain Boundaries in Metals. Moscow, Metallurgy Publ., 1980, 224 p. (in Russ.) 
7. Gleyter G., Chalmers B. High-angle Grain Boundaries. Moscow, Mir Publ., 1975, 376 p. (in Russ.)

8. Straumal B. B., Shvindlerman H. P. Surface. Physics, Chemistry, Mechanics, 1986, vol. 10, pp. 5-14. (in Russ.)

9. Fortes M. A. Phys. Stat. Sol., 1977, vol. B 82, pp. 377382. DOI: https://doi.org/10.1002/pssb. 2220820143

10. Bonnet R., Durand F. Scripta Met., 1975, vol. 9, pp. 935-939. DOI: https://doi.org/10.1016/00369748(75)90548-7

11. Bonnet R. Scripta Met., 1976, vol. 10, pp. 801-806. DOI: https://doi.org/10.1016/0036-9748(76)90297-0

12. Bonnet R., Cousineau E. Acta Cryst., 1977, vol. A 33, pp. 850-856. DOI: https://doi.org/10.1107/ S0567739477002058

13. Rybin V. V., Perevezentsev V. N. FTT [Technical Physics], 1975, vol. 17, pp. 3188-3193. (in Russ.)

14. Andreeva A. V., Fionova L. K. Fizika Metallov $i$ Metallovedenie [Physics of Metals and Metallography], 1977, vol. 44, pp. 395-400. (in Russ.)
15. Kaibyshev O. A., Valiev R. Z. Grain Boundaries and Properties of Metals. Moscow, Metallurgy Publ., 1987, 214 p. (in Russ.)

16. Kopetsky H. V., Orlov A. N., Fionova L. K. Grain Boundaries in Pure Materials. Moscow, Nauka Publ., 1987, 160 p. (in Russ.)

17. Bokshtein B. S. Structure and Properties of Internal Interfaces in Metals. Moscow, Metallurgy Publ., 1988, 272 p. (in Russ.)

18. Kobayashi S., Tsurekawa S., Watanabe T. Beilstein J. Nanotechnol, 2016, vol. 7, pp. 1829-1849. DOI: https:// doi.org/10.3762/bjnano.7.176

19. Sukhomlin G. D. Metallofizika, noveishie tekhnologii, 2013, vol. 35, iss. 9, pp. 1237-1249. (in Russ.)

20. Watanabe T. Journal of Materials Science, 2011, vol. 46, pp 4095-4115. DOI: https://doi.org/10.1007/s10853011-5393-z
Даринский Борис Михайлович - д. ф.-м. н., профессор, профессор кафедры материаловедения и индустрии наносистем, Воронежский государственный университет, Воронеж, Россия; тел.: +7 (473) 2208735, e-mail: darinskii@mail.ru

Ефанова Наталия Дмитриевна - студент физического факультета, Воронежский государственный университет, Воронеж, Россия; тел.: +7 (950) 7585719, e-mail: efanowanatalia@gmail.com

Прижимов Андрей Сергеевич - к. ф.-м. н., доцент кафедры материаловедения и индустрии наносистем, Воронежский государственный университет, Воронеж, Россия; тел.: +7 (473) 2208735, e-mail: rnileme@mail.ru
Boris M. Darinskiy - Dr. Sci. (Phys.-Math.), Full Professor, Voronezh State University, Voronezh, Russia; tel.:+7 (473) 2208735, e-mail: darinskii@ mail.ru

Natalia D. Efanova - Student of Physics Faculty, Voronezh State University, Voronezh, Russia; tel.: +7 (950) 7585719, e-mail: efanowanatalia@gmail. com

Andrey S. Prizhimov - Cand. Sci. (Phys.-Math.), Senior Researcher, Voronezh State University, Voronezh, Russia; tel.:+7 (473) 2208735, e-mail: rnileme@mail.ru 